\title{
On the geometry of higher-spin gauge fields
}

\author{
Dario Francia* and Augusto Sagnotti ${ }^{\dagger}$ \\ Dipartimento di Fisica, Univ. Roma "Tor Vergata" and I.N.F.N., Sezione di Roma \\ "Tor Vergata" \\ Via della Ricerca Scientifica, 100133 Roma ITALY \\ E-mail: sagnotti@roma2.infn.it
}

\begin{abstract}
We review a recent construction of the free field equations for totally symmetric tensors and tensor-spinors that exhibits the corresponding linearized geometry. These equations are not local for all spins $>2$, involve unconstrained fields and gauge parameters, rest on the curvatures introduced long ago by de Wit and Freedman, and reduce to the local (Fang-)Fronsdal form upon partial gauge fixing. We also describe how the higher-spin geometry is realized in free String Field Theory, and how the gauge fixing to the light cone can be effected. Finally, we review the essential features of local compensator forms for the higher-spin bosonic and fermionic equations with the same unconstrained gauge symmetry.
\end{abstract}

\section{Introduction}

This article reviews the results of [1], 2] on the geometry underlying the free equations for symmetric tensors of arbitrary spin $s$ and on its role in corresponding equations for fermionic tensors of spin $s+1 / 2$. It is meant as a contribution to the Conference Proceedings of the Sakharov, Leuven and SIGRAV Meetings, and to the Proceedings of the 2002 Cargese Summer Institute. The SIGRAV contribution was accompanied by a less technical lecture at the companion SIGRAV School, essentially based on [3], while only one of the three Cargese lectures was actually devoted to this topic. However, since the other two dealt with results reviewed in detail in [⿶, it will suffice to confine the attention to this topic. This will also provide an opportunity to supplement the results in [1] with additional remarks on their role in String Theory and on the issue of gauge fixing ${ }^{1}$. The last two sections, added in this new version, review the results in 2 and present local compensator forms

\footnotetext{
${ }^{*}$ Present address: Dipartimento di Fisica, Università di Roma Tre and INFN, Sezione di Roma Tre, Via della Vasca Navale 84, 00146 Roma ITALY.

${ }^{\dagger}$ Speaker.

${ }^{1}$ We are grateful to A. Segal for his stimulating remarks on this issue.
} 
for the higher-spin bosonic and fermionic equations with the same unconstrained gauge symmetry.

The starting point for our discussion are the elegant equations extracted long ago by Fronsdal [5] from the massive Singh-Hagen [6] actions,

$$
\mathcal{F} \equiv \square \phi-\partial \partial \cdot \phi+\partial^{2} \phi^{\prime}=0
$$

that for spin one and two reduce to the Maxwell equation for the vector potential $A_{\mu}$ and to the linearized Einstein equation for the metric fluctuation $h_{\mu \nu}$. Here, as will often be the case in the following, primes (or bracketed suffixes) denote traces, while all indices carried by the symmetric tensors $\phi_{\mu_{1} \ldots \mu_{s}}$ and $\eta_{\mu \nu}$ or by derivatives are left implicit. In this shorthand notation, where all terms are meant to be totally symmetrized, one need only get accustomed to a few rules, some notable examples for a $D$-dimensional space time being

$$
\begin{aligned}
& \left(\partial^{p} \phi\right)^{\prime}=\square \partial^{p-2} \phi+2 \partial^{p-1} \partial \cdot \phi+\partial^{p} \phi^{\prime}, \\
& \partial^{p} \partial^{q}=\left(\begin{array}{c}
p+q \\
p
\end{array}\right) \partial^{p+q}, \\
& \partial \cdot\left(\partial^{p} \phi\right)=\square \partial^{p-1} \phi+\partial^{p} \partial \cdot \phi, \\
& \partial \cdot \eta^{k}=\partial \eta^{k-1}, \\
& \left(\eta^{k} T_{(s)}\right)^{\prime}=k[D+2(s+k-1)] \eta^{k-1} T_{(s)}+\eta^{k} T_{(s)}^{\prime},
\end{aligned}
$$

where $T_{(s)}$ denotes a symmetric rank-s tensor, but the general case can then be dealt with in a rather efficient and compact fashion.

At a closer look, however, for spin $s>2$ eq. (1.1) appears somewhat less natural than its first and most familiar instances,

$$
\begin{aligned}
& \square A_{\mu}-\partial_{\mu} \partial \cdot A=0, \\
& \square h_{\mu \nu}-\partial_{\mu} \partial \cdot h_{\nu}-\partial_{\nu} \partial \cdot h_{\mu}+\partial_{\mu} \partial_{\nu} h^{\prime}=0,
\end{aligned}
$$

since only these involve all available lower spin constructs built from divergences and traces of the gauge field. This peculiarity has in fact an important consequence: only for spin one and two does the gauge transformation

$$
\delta \phi=\partial \Lambda
$$

leave the Fronsdal operator $\mathcal{F}$ invariant, while in general

$$
\delta \mathcal{F}=3 \partial^{3} \Lambda^{\prime},
$$

and as a result for spin $s \geq 3$ eq. (1.1) requires a traceless gauge parameter $\Lambda$. This constraint plays a key role in the Fronsdal formulation [5], together with an additional one, effective from $s=4$ onward, on the gauge field, whose double trace $\phi^{\prime \prime}$ is also required to vanish.

We can now review how one can actually forego both constraints, at the price of allowing non-local terms both in the free field equations and in the corresponding Lagrangians, 
but with the clear advantage of eliciting the geometry that underlies them. This geometry would be naturally expected to play a role in the Vasiliev equations 17 , 8, but to date we have not gone far enough to make definite statements to this effect. However, it is a fact that String Field Theory [9, 10], in the most symmetric formulation of its free field equations, deduced in [10] from the BRST analysis of [11], does not involve trace conditions on the gauge fields or on the corresponding gauge parameters. Rather, as we shall see, it embodies very nicely the higher-spin geometric equations that we are about to review.

Let us therefore begin by tracing the origin of the double trace constraint on the gauge field. To this end, it is important to recall that, already at spin two, the naive linearized Einstein equation

$$
R_{\mu \nu}^{(l i n)}=0
$$

does not follow directly from the Einstein-Hilbert action principle, whose variation actually gives

$$
\delta \mathcal{L} \sim \delta h_{\mu \nu}\left[R_{\mu \nu}^{(l i n)}-\frac{1}{2} \eta_{\mu \nu} R^{(l i n)}\right] .
$$

There is a clear logic behind this result, however, since the linearized Einstein tensor

$$
G_{\mu \nu}^{(l i n)}=R_{\mu \nu}^{(l i n)}-\frac{1}{2} \eta_{\mu \nu} R^{(l i n)}
$$

has the special virtue of being divergence free, and this ensures that $\delta L$ vanishes, up to a total derivative, if $\delta h^{\mu \nu}$ corresponds to a gauge transformation. In other words, the gauge invariance of $L$ rests on two ingredients: the gauge invariance of the field equation and a suitable Bianchi identity. This pattern extends to all higher-spin symmetric tensors, whose Bianchi identities

$$
\partial \cdot \mathcal{F}-\frac{1}{2} \partial \mathcal{F}^{\prime}=-\frac{3}{2} \partial^{3} \phi^{\prime \prime}
$$

however, contain a sort of classical anomaly that involves their double traces. As a result, for spin $>2$ the Einstein-like tensors

$$
\mathcal{G}=\mathcal{F}-\frac{1}{2} \eta \mathcal{F}^{\prime}
$$

can only be compatible with gauge invariant Lagrangians such that

$$
\delta \mathcal{L} \sim \delta \phi\left[\mathcal{F}-\frac{1}{2} \eta \mathcal{F}^{\prime}\right]
$$

if the gauge parameters $\Lambda$ of eq. (1.9) are traceless and the gauge fields $\phi$ are doubly traceless. Using the combinatoric rules listed above one can indeed show that, up to a total derivative, the gauge transformation (1.9) turns eq. (1.16) into

$$
\delta \mathcal{L} \sim \Lambda\left[\partial \cdot \mathcal{F}-\frac{1}{2} \partial \mathcal{F}^{\prime}-\frac{1}{2} \eta \partial \cdot \mathcal{F}^{\prime}\right]
$$

that can only vanish in general if both restrictions are enforced. Notice that the Bianchi identity eliminates the first two terms, while the last does not vanish identically. Rather, via the $\eta$ tensors, it places again on the gauge parameter the same restriction that ensures 
the gauge invariance of the Fronsdal operator $\mathcal{F}$. To reiterate, the restriction to traceless gauge parameters is instrumental in the gauge invariance of the Fronsdal equations, while the restriction to doubly traceless fields ensures that the corresponding action principles be compatible with the gauge symmetry.

\section{Gauge-invariant equations for unconstrained bosons}

In order to forego these restrictions, one can follow an iterative procedure that, as in 罒, can be conveniently motivated in the relatively simple spin-3 case, where

$$
\delta \mathcal{F}_{\mu_{1} \mu_{2} \mu_{3}}=3 \partial_{\mu_{1}} \partial_{\mu_{2}} \partial_{\mu_{3}} \Lambda^{\prime}
$$

since one can find rather simply other non-local constructs of $\mathcal{F}$ that vary as in (2.1), to wit

$$
\begin{aligned}
& \frac{1}{3 \square}\left[\partial_{\mu_{1}} \partial_{\mu_{2}} \mathcal{F}^{\prime}{ }_{\mu_{3}}+\partial_{\mu_{2}} \partial_{\mu_{3}} \mathcal{F}_{\mu_{1}}+\partial_{\mu_{3}} \partial_{\mu_{1}} \mathcal{F}^{\prime}{ }_{\mu_{2}}\right], \\
& \frac{1}{3 \square}\left[\partial_{\mu_{1}} \partial \cdot \mathcal{F}_{\mu_{2} \mu_{3}}+\partial_{\mu_{2}} \partial \cdot \mathcal{F}_{\mu_{3} \mu_{1}}+\partial_{\mu_{3}} \partial \cdot \mathcal{F}_{\mu_{1} \mu_{2}}\right], \\
& \frac{1}{\square^{2}} \partial_{\mu_{1}} \partial_{\mu_{2}} \partial_{\mu_{3}} \partial \cdot \mathcal{F}^{\prime} .
\end{aligned}
$$

The first two expressions actually coincide, as can be seen making use of the Bianchi identity (1.14), and as a result one is led to two apparently distinct forms for a non-local fully gauge invariant field equation:

$$
\begin{aligned}
& \mathcal{F}_{\mu_{1} \mu_{3} \mu_{3}}-\frac{1}{3 \square}\left[\partial_{\mu_{1}} \partial_{\mu_{2}} \mathcal{F}^{\prime}{ }_{\mu_{3}}+\partial_{\mu_{2}} \partial_{\mu_{3}} \mathcal{F}^{\prime}{ }_{\mu_{1}}+\partial_{\mu_{3}} \partial_{\mu_{1}} \mathcal{F}^{\prime}{ }_{\mu_{2}}\right]=0 \\
& \mathcal{F}_{\mu_{1} \mu_{2} \mu_{3}}-\frac{1}{\square^{2}} \partial_{\mu_{1}} \partial_{\mu_{2}} \partial_{\mu_{3}} \partial \cdot \mathcal{F}^{\prime}=0
\end{aligned}
$$

These can be actually turned into one another, once they are combined with their traces, but the second form is clearly somewhat simpler, since its rests on the addition of the spin- 0 construct $\partial \cdot \mathcal{F}^{\prime}$.

For all higher spins, one can arrive at the proper analogue of (2.3) via a sequence of pseudo-differential operators, defined recursively as

$$
\mathcal{F}^{(n+1)}=\mathcal{F}^{(n)}+\frac{1}{(n+1)(2 n+1)} \frac{\partial^{2}}{\square} \mathcal{F}^{(n) \prime}-\frac{1}{n+1} \frac{\partial}{\square} \partial \cdot \mathcal{F}^{(n)},
$$

where $\mathcal{F}^{(1)}=\mathcal{F}$, whose gauge transformations,

$$
\delta \mathcal{F}^{(n)}=(2 n+1) \frac{\partial^{2 n+1}}{\square^{n-1}} \Lambda^{[n]},
$$

involve by construction higher traces of the gauge parameter. Since the $n$-th trace $\Lambda^{[n]}$ is only available for spins $s \geq 2 n+1$, after a certain number of iterations one is bound to end up with a fully gauge invariant, albeit non-local, kinetic operator. 
A similar inductive argument determines the Bianchi identities for the $\mathcal{F}^{(n)}$,

$$
\partial \cdot \mathcal{F}^{(n)}-\frac{1}{2 n} \partial \mathcal{F}^{(n) \prime}=-\left(1+\frac{1}{2 n}\right) \frac{\partial^{2 n+1}}{\square^{n-1}} \phi^{[n+1]},
$$

where the "anomalous" contribution depends on the $(n+1)$-th trace $\phi^{[n+1]}$ of the gauge field, and thus vanishes for all spins $s<2 n+2$. Summarizing, these results imply that, after a certain number of iterations, one can obtain non-local Lagrangians and field equations whose gauge invariance places no restrictions on the gauge fields or on the corresponding gauge parameters. Eq. (2.7) also implies similar relations for the higher traces of the $\mathcal{F}^{(n)}$,

$$
\partial \cdot \mathcal{F}^{(n)[k]}-\frac{1}{2(n-k)} \partial \mathcal{F}^{(n)[k+1]}=0, \quad(k \leq n-1)
$$

here written for $n$ large enough so that the "anomaly" on the r.h.s. of (2.7) vanishes identically. Notice that for odd $\operatorname{spin} s=2 n-1$ the second term vanishes for the last trace, so that

$$
\partial \cdot \mathcal{F}^{(n)[n-1]}=0 .
$$

These generalized Bianchi identities suffice to define for all spin- $s$ fields fully gauge invariant analogues of the Einstein tensor,

$$
\mathcal{G}^{(n)}=\sum_{p \leq n} \frac{(-1)^{p}}{2^{p} p !\left(\begin{array}{l}
n \\
p
\end{array}\right)} \eta^{p} \mathcal{F}^{(n)[p]},
$$

obtained combining the proper $\mathcal{F}^{(n)}$ with their multiple traces that, for $n$ large enough, have vanishing divergences like their spin-2 counterpart. This is attained directly by the subtractions for all even spins, while for odd spins the last term thus generated vanishes on account of (2.9). From the $\mathcal{G}^{(n)}$ tensors, integrating

$$
\delta \mathcal{L} \sim \delta \phi \mathcal{G}^{(n)}
$$

one can then recover the corresponding gauge invariant Lagrangians.

In order to write the fully gauge invariant equations in a compact form, it is convenient to resort to the trick of [15], contracting the gauge fields $\phi_{\mu_{1} \cdots \mu_{s}}$ with a vector $\xi$. It is then simple to show that traces and divergences of the resulting expressions,

$$
\hat{\Phi}(x, \xi)=\frac{1}{s !} \xi^{\mu_{1}} \cdots \xi^{\mu_{s}} \phi_{\mu_{1} \cdots \mu_{s}},
$$

can be recovered applying the differential operators $\partial_{\xi} \cdot \partial_{\xi}$ and $\partial_{\xi} \cdot \partial$, where $\partial_{\xi}$ denotes a derivative with respect to $\xi$. Starting from the Fronsdal term

$$
\hat{\mathcal{F}}(\hat{\Phi})=\left[\square-\xi \cdot \partial \partial \cdot \partial_{\xi}+(\xi \cdot \partial)^{2} \partial_{\xi} \cdot \partial_{\xi}\right] \hat{\Phi},
$$

the result of the successive iterations can then be written in the compact form

$$
\prod_{k=0}^{n-1}\left[1+\frac{(\xi \cdot \partial)^{2}}{(k+1)(2 k+1)} \frac{\partial_{\xi} \cdot \partial_{\xi}}{\square}-\frac{\xi \cdot \partial}{k+1} \frac{\partial_{\xi} \cdot \partial}{\square}\right] \hat{\mathcal{F}}(\hat{\Phi})=0
$$


where for spin $s$ a fully gauge invariant operator is reached after $\left[\frac{s+1}{2}\right]$ iterations. Expanding this expression and combining it with its trace it is then possible to show that the field equations can always be reduced to the form

$$
\mathcal{F}=\partial^{3} \mathcal{H}
$$

that generalizes eq. (2.4). Under a gauge transformation, by consistency, $\delta \mathcal{H}=3 \Lambda^{\prime}$, and therefore the local forms (2.13) can be recovered from (2.14) making use of the traces of the gauge parameters $\Lambda$ to reach the "Fronsdal gauges" $\mathcal{H}=0$. The final gauge fixing to the light cone, however, presents some subtleties, and will be discussed in a later section.

Gauge invariant Lagrangians can be also associated to eq. (2.15), as can already be seen for spin 3. They differ from those corresponding to the $\mathcal{F}^{(n)}$, and have the virtue of reducing to the local expressions of [5] in the Fronsdal gauges $\mathcal{H}=0$, but the general expressions for the corresponding Einstein-like tensors are less transparent, and we shall thus refrain from displaying them explicitly.

\section{Gauge-invariant equations for unconstrained fermions}

One can also arrive at similar non-local geometric equations for totally symmetric tensorspinors $\psi_{\mu_{1} \cdots \mu_{s}}$. In this case the local fermionic equations of [0]

$$
\mathcal{S} \equiv i(\not \partial \psi-\partial \psi)=0
$$

are gauge invariant under

$$
\delta \psi=\partial \epsilon
$$

only if the gauge parameters are subject to the constraint

$$
\notin=0 \text {. }
$$

In addition, the $\mathcal{S}$ operators satisfy the "anomalous" Bianchi identities

$$
\partial \cdot \mathcal{S}-\frac{1}{2} \partial \mathcal{S}^{\prime}-\frac{1}{2} \not \partial \phi=i \partial^{2} \psi^{\prime}
$$

and therefore the gauge variation of the generic Lagrangian

$$
\delta \mathcal{L} \sim \delta \bar{\psi}\left[\mathcal{S}-\frac{1}{2}\left(\eta \mathcal{S}^{\prime}+\gamma \not \mathcal{S}\right)\right]
$$

can only vanish if eq. (3.3) is supplemented by

$$
\psi^{\prime}=0,
$$

the fermionic analogue of the double trace condition for boson fields.

It is convenient to notice that the fermionic operators for spin $s+1 / 2$ can be formally related to the bosonic operators for $\operatorname{spin} s$ according to

$$
\mathcal{S}_{s+1 / 2}-\frac{1}{2} \frac{\partial}{\square} \not \partial \mathcal{S}_{s+1 / 2}=i \frac{\not \partial}{\square} \mathcal{F}_{s}(\psi) .
$$


This amusing link generalizes the obvious one between the Dirac and Klein- Gordon operators and, for the rather familiar $s=1$ case, connects the Rarita-Schwinger operator, that when combined with its $\gamma$ trace can be reduced to the form (3.1), to the Maxwell operator, according to

$$
\mathcal{S}_{\mu}-\frac{1}{2} \frac{\partial_{\mu} \not \partial}{\square} \not=i \frac{\not \partial}{\square}\left[\square \eta_{\mu \nu}-\partial_{\mu} \partial_{\nu}\right] A^{\nu}
$$

where we have restored the conventional index notation.

One can also show, by an inductive argument, that similar relations hold between the non-local fully gauge invariant counterparts of eqs. (1.1) and (3.1). They link the fermionic kinetic operators $\mathcal{S}^{(n)}$, defined recursively as

$$
\mathcal{S}^{(n+1)}=\mathcal{S}^{(n)}+\frac{1}{n(2 n+1)} \frac{\partial^{2}}{\square} \mathcal{S}^{(n)^{\prime}}-\frac{2}{2 n+1} \frac{\partial}{\square} \partial \cdot \mathcal{S}^{(n)}
$$

and such that

$$
\delta \mathcal{S}^{(n)}=-2 i n \frac{\partial^{2 n}}{\square^{n-1}} \phi^{[n-1]},
$$

to the corresponding corrected bosonic operators of the previous section, according to

$$
\mathcal{S}_{s+1 / 2}^{(n)}-\frac{1}{2 n} \frac{\partial}{\square} \not \partial \mathcal{S}_{s+1 / 2}^{(n)}=i \frac{\not \partial}{\square} \mathcal{F}_{s}^{(n)}(\psi) .
$$

This relation also determines the "anomalous" Bianchi identities of the $\mathcal{S}^{(n)}$,

$$
\partial \cdot \mathcal{S}^{(n)}-\frac{1}{2 n} \partial \mathcal{S}^{(n)^{\prime}}-\frac{1}{2 n} \not \partial \mathcal{S}^{(n)}=i \frac{\partial^{2 n}}{\square^{n-1}} \psi^{[n]},
$$

and therefore, for $n$ large enough so that $S^{(n)}$ is gauge invariant and eq. (3.12) is free of the "anomalous" term, the corrected Einstein-like operators

$$
\mathcal{G}^{(n)}=\mathcal{S}^{(n)}+\sum_{0<p \leq n} \frac{(-1)^{p}}{2^{p} p !\left(\begin{array}{l}
n \\
p
\end{array}\right)} \eta^{p-1}\left[\eta \mathcal{S}^{(n)[p]}+\gamma \mathcal{S}^{(n)[p-1]}\right],
$$

whose divergences vanish on account of eqs. (3.12) and of their $(\gamma-)$ traces, and from which gauge invariant Lagrangians can be recovered. For the sake of brevity, in the following we shall mostly restrict our attention to bosonic higher-spin fields.

\section{Free-field geometry}

Following de Wit and Freedman [16], one can define generalized connections of various orders in the derivatives for all spin- $s$ gauge fields. This can be done by an iterative procedure, so that, in the compact notation of the previous section, for any field of spin $s$, after $m$ iterations one can define

$$
\Gamma^{(m)}=\frac{1}{m+1} \sum_{k=0}^{m} \frac{(-1)^{k}}{\left(\begin{array}{c}
m \\
k
\end{array}\right)} \partial^{m-k} \nabla^{k} \varphi,
$$

where we are now using two types of derivatives for two distinct sets of indices, $\partial$ for the $m$ symmetric indices $\left(\alpha_{1} \cdots \alpha_{m}\right)$ introduced by the derivatives and $\nabla$ for the other $s$ 
symmetric ones $\left(\beta_{1} \cdots \beta_{s}\right)$ originating from the gauge field. It is simple to show, by an inductive argument, that the gauge transformation of $\Gamma^{(m)}$ is

$$
\delta \Gamma^{(m)}=\nabla^{m+1} \Lambda
$$

so that all $m$ indices of the first set are within the gauge parameter. Hence,

$$
\Gamma^{(s-1)}=\frac{1}{s} \sum_{k=0}^{s-1} \frac{(-1)^{k}}{\left(\begin{array}{c}
s-1 \\
k
\end{array}\right)} \partial^{s-k-1} \nabla^{k} \phi,
$$

is the proper analogue of the Christoffel connection for a spin- $s$ gauge field, since its gauge transformation contains a single term. That these objects can be defined in general can be also recognized noticing that the $(s-1)$-th derivatives of the spin- $s$ gauge transformations of eq. (1.9) imply that one can retrieve a composite connection $\Gamma_{\alpha_{1} \cdots \alpha_{s-1} ; \beta_{1} \cdots \beta_{s}}$, such that

$$
\delta \Gamma_{\alpha_{1} \cdots \alpha_{s-1} ; \beta_{1} \cdots \beta_{s}}=\partial_{\beta_{1}} \cdots \partial_{\beta_{s}} \Lambda^{\alpha_{1} \cdots \alpha_{s-1}}
$$

inverting a linear system with $\left(\begin{array}{c}2 s-1 \\ s\end{array}\right)$ unknowns. Moreover, all $\Gamma$ 's with $m>s$ are gauge invariant, and in particular

$$
\Gamma^{(s)}=\frac{1}{s+1} \sum_{k=0}^{s} \frac{(-1)^{k}}{\left(\begin{array}{l}
s \\
k
\end{array}\right)} \partial^{s-k} \nabla^{k} \phi
$$

is the proper analogue of the Riemann curvature tensor. This generalized curvature is of the form $\mathcal{R}_{\alpha_{1} \cdots \alpha_{s} ; \beta_{1} \cdots \beta_{s}}$, and is totally symmetric under the interchange of any pair of indices within each of the two sets. In addition, as shown in [16],

$$
\mathcal{R}_{\alpha_{1} \cdots \alpha_{s} ; \beta_{1} \cdots \beta_{s}}=(-1)^{s} \mathcal{R}_{\beta_{1} \cdots \beta_{s} ; \alpha_{1} \cdots \alpha_{s}}
$$

and a generalized cyclic identity holds. These concepts can be also related to an interesting generalization of the exterior differential, whereby the familiar condition $d^{2}=0$ is replaced by $d^{s+1}=0$ 17.

There is another, perhaps more obvious way, to generate a gauge invariant quantity from a connection $\Gamma^{(s-1)}$ that transforms as in (4.4), a curl with respect to any of its $\beta$ indices. However, the choice of 16] has the virtue of simplicity, since it results automatically in a tensor with two totally symmetric sets of indices. For instance, the symmetric curvature of [16] for the spin-2 case is a linear combination of ordinary Riemann tensors, while its trace in the first symmetric set of indices is proportional to the ordinary Ricci tensor. If we now restrict our attention to the $\Gamma^{(m)}$ 's with $m$ even, and for the sake of clarity let $m=2 n$, eq. (4.2) implies that the total trace of $\Gamma^{(2 n)}$ over pairs of $\beta$ indices, $\Gamma^{(2 n)[n]}$, is a totally symmetric spin- $s$ tensor such that

$$
\delta \frac{1}{\square^{n-1}} \Gamma^{(2 n)[n]}=\frac{\partial^{2 n+1}}{\square^{n-1}} \Lambda^{[n]} .
$$

Up to an overall proportionality constant, this is exactly the gauge transformation of the $\mathcal{F}^{(n)}$, and in particular, if $s=2 n, \Gamma^{(2 n)[n]}$ is the $n$-th trace of the spin- $s$ analogue of 
the Riemann tensor defined above. The iterative procedure of the previous section is thus providing a rôle for the higher-spin connections of [16], so that the geometric gaugeinvariant equations for even spin $s=2 n$ can be written in the form

$$
\frac{1}{\square^{n-1}} \mathcal{R}_{; \mu_{1} \cdots \mu_{2 n}}^{[n]}=0
$$

a natural generalization of the linearized Einstein equation.

The odd-spin case $s=2 n+1$ presents a further minor subtlety, in that the corresponding curvatures $\Gamma^{2 n+1}$ have an odd number of $\beta$ indices. The simplest option is then to take a trace over $n$ pairs of $\beta$ indices in $\Gamma^{2 n+1}$ and act with a divergence on the remaining one. The end result for spin $s=2 n+1$ is then

$$
\frac{1}{\square^{n}} \partial \cdot \mathcal{R}_{; \mu_{1} \cdots \mu_{2 n+1}}^{[n]}=0
$$

a natural generalization of the Maxwell equation. Notice that the Maxwell and Einstein cases are the only ones when these geometric equations are local. In all cases, however, the Fronsdal operators provide local, albeit partly gauge fixed, forms for them. As anticipated in the previous sections, these are the least singular fully gauge invariant equations, while more singular forms can be obtained combining eqs. (4.8) and (4.9) with their traces, as in section 2 .

\section{The issue of gauge fixing}

In the previous sections we have seen how the non-local geometric equations for higher-spin bosons can be reduced to the usual local forms using the traces $\Lambda^{\prime}$ of the gauge parameters to reach the "Fronsdal gauges" $\mathcal{H}=0$. The resulting Fronsdal equations, however, present a subtlety, since they involve unconstrained fields $\phi$, whose double traces $\phi^{\prime \prime}$ do not vanish identically. As we shall see, this subtlety requires a slight revision of the standard gaugefixing procedure and, as a result, the field equations themselves set to zero the double traces on shell, leaving again the right set of modes.

Whereas in the usual local formulation the de Donder gauge condition

$$
\mathcal{D} \equiv \partial \cdot \phi-\frac{1}{2} \partial \phi^{\prime}=0
$$

is used to reduce the Fronsdal equation to the canonical form

$$
\square \phi=0,
$$

the same procedure runs into a difficulty in this case. Indeed, while the gauge variation of $\mathcal{D}$ under (1.9) is in general

$$
\delta \mathcal{D}=\square \Lambda-\partial^{2} \Lambda^{\prime}
$$

once the trace of the gauge parameter has been used to set $\mathcal{H}=0$, eq. (5.3) can only be consistent if the de Donder gauge condition is modified in order that it be identically traceless. While not automatic, since in our case $\phi^{\prime \prime}$ does not vanish identically as in [5], 
this can be achieved following once more an iterative procedure. Before dealing with the general case, it is perhaps instructive to discuss explicitly this issue for a spin- 4 field, the first case where the problem presents itself.

For a spin- 4 field in $D$ dimensions, one can modify $\mathcal{D}$ by the addition of a higher trace, to finally impose

$$
\mathcal{D}+\Delta \equiv \partial \cdot \phi-\frac{1}{2} \partial \phi^{\prime}+\frac{1}{2(D+2)} \eta \partial \phi^{\prime \prime}=0
$$

that is identically traceless. When used in the Fronsdal equation, in momentum space this leads to

$$
p^{2} \phi_{\mu \nu \rho \sigma}+\frac{1}{D+2}\left[\eta_{\mu \nu} p_{\rho} p_{\sigma}+\cdots\right] \phi^{\prime \prime}=0
$$

and this equation can only have non-trivial eigentensors if $p^{2}=0$, since the second term does not contain any dimensionful parameter. With a light-like momentum $p_{+}$, choosing for instance $(\mu, \nu, \rho, \sigma)$ equal to $(+,-,+,+)$, it is then simple to see that eq. (5.5) sets $\phi^{\prime \prime}=0$. At this point, the gauge condition reduces to the usual de Donder form, with a field now constrained as in [5]. The non-local equation thus propagates correctly spin4 polarizations, despite the fact that a traceless gauge parameter, left over after setting $\mathcal{H}=0$, is originally accompanied by an unconstrained gauge field.

In general, for a spin- $s$ field $\phi$ one can define

$$
\Delta=\sum_{k \geq 2}\left[c_{k, 1} \eta^{k-1} \partial \phi^{[k]}+c_{k, 2} \eta^{k} \partial \cdot \phi^{[k]}\right]
$$

where the coefficients $c_{k, 1}$, available for $k \leq\left[\frac{s}{2}\right]$, and $c_{k, 2}$, available $k \leq\left[\frac{s-1}{2}\right]$, can be determined recursively requiring that

$$
\begin{aligned}
\Delta^{\prime}=\sum_{k \geq 2}\{ & \eta^{k-1} \partial \cdot \phi^{[k]}\left[2 c_{k, 1}+c_{k, 2} k(D+2(s-k-2))\right] \\
& +c_{k, 1}(k-1)(D+2(s-k-1)) \eta^{k-2} \partial \phi^{[k]} \\
& \left.+c_{k, 1} \eta^{k-1} \partial \phi^{[k+1]}+c_{k, 2} \eta^{k} \partial \cdot \phi^{[k+1]}\right\}
\end{aligned}
$$

cancel the trace of the naive de Donder condition $\mathcal{D}$, i.e. that

$$
\Delta^{\prime}-\frac{1}{2} \partial \phi^{\prime \prime}=0
$$

so that

$$
\mathcal{D}+\Delta=0
$$

is an allowed gauge condition. This requires that

$$
c_{k+1,1}=-\frac{c_{k, 1}}{k[D+2(s-k-2)]},
$$


and therefore

$$
c_{k, 1}=\frac{(-1)^{k}}{2(k-1) ! \prod_{2 \leq \ell \leq k}[D+2(s-\ell-1)]} \quad(k \geq 2) .
$$

The $c_{k, 2}$ are then determined by the inhomogeneous difference equation

$$
c_{k+1,2}+\frac{c_{k, 2}}{(k+1)[D+2(s-k-3)]}=g_{k}
$$

where

$$
g_{k}=\frac{(-1)^{k}}{2(k+1) ! \prod_{2 \leq \ell \leq(k+2)}[D+2(s-\ell-1)]},
$$

whose solution is

$$
c_{k, 2}=\frac{(-1)^{k-1}}{2(k-1) ! \prod_{2 \leq \ell \leq(k+1)}[D+2(s-\ell-1)]} \quad(k \geq 2) .
$$

As a result, the field equations reduce in general to

$$
\square \phi+\partial \Delta=0,
$$

while the existence of non-vanishing solutions requires that $p^{2}=0$, the only covariant condition that can emerge from a kinetic operator, invertible as a result of the gauge-fixing procedure, that only involves dimensionless parameters. Eqs. (5.9) then turn (5.15) into

$$
\partial \mathcal{D}=0
$$

and, with a light-like momentum $p_{+}$, it is easy to convince oneself that these imply

$$
\mathcal{D}=0
$$

and therefore, via their traces, $\phi^{\prime \prime}=0$.

When combined, as usual, with residual gauge transformations, the de Donder conditions thus retrieved leave only the polarizations corresponding to irreducible, traceless symmetric spin- $s$ tensors. Still, the need for these generalized $(\mathcal{D}+\Delta)$-gauges appears to reflect interesting properties of this formulation at the quantum level, that deserve further investigation.

\section{Higher-spin geometry and String Theory}

We now want to show that the higher-spin geometry discussed in the previous sections plays a clear, albeit indirect, role in String Theory. That this should be the case can be simply anticipated. Following [11, 10], the free equations of String Field Theory can indeed be written in the form

$$
\mathcal{Q}|\Phi\rangle=0,
$$

where $\mathcal{Q}$ denotes the BRST operator of the first-quantized string, and have the chain of gauge invariances

$$
\delta\left|\Phi^{(n)}\right\rangle=\mathcal{Q}\left|\Phi^{(n+1)}\right\rangle,
$$


with $\Phi^{(1)}$ the string gauge field and $\Phi^{(n)}(n>1)$ the corresponding chain of gauge (per gauge) parameters. It should be appreciated that neither (6.1) nor (6.2) involve trace conditions. Whereas they describe massive higher-spin modes, their limit as $\alpha^{\prime} \rightarrow \infty$ is thus expected to result in fully gauge invariant free massless higher-spin equations with no trace constraints like those in [5. This is indeed the case, and for symmetric tensors (say, in the "leading Regge trajectory" generated by the lowest string oscillators $\alpha_{-1}$ in the bosonic string), the limit $\alpha^{\prime} \rightarrow \infty$ yields the equations

$$
\begin{aligned}
& \square \phi=\partial C, \\
& \partial \cdot \phi-\partial D=C, \\
& \square D=\partial \cdot C,
\end{aligned}
$$

that link the unconstrained spin-s tensors $\phi$, spin- $(s-1)$ tensors $C$ and spin- $(s-2)$ tensors $D$, are invariant under the unconstrained gauge transformations

$$
\begin{aligned}
& \delta \phi=\partial \Lambda, \\
& \delta C=\square \Lambda, \\
& \delta D=\partial \cdot \Lambda,
\end{aligned}
$$

and follow from the Lagrangians

$$
\begin{aligned}
\mathcal{L}= & -\frac{1}{2} \partial_{\mu} \phi \partial^{\mu} \phi-\phi \partial C-s C \partial D \\
& -\frac{s}{2} C^{2}-\left(\begin{array}{l}
s \\
2
\end{array}\right) \partial_{\mu} D \partial^{\mu} D .
\end{aligned}
$$

On can also eliminate the auxiliary field $C$, reducing eqs. (6.3) to the pair of equations

$$
\begin{aligned}
& \mathcal{F}=\partial^{2}\left(\varphi^{\prime}-2 D\right), \\
& \square D=\frac{1}{2} \partial \cdot \partial \cdot \varphi-\frac{1}{2} \partial \partial \cdot D,
\end{aligned}
$$

One can also introduce similar fermionic systems,

$$
\begin{aligned}
& \not \partial \psi=\partial \chi, \\
& \partial \cdot \psi-\partial \lambda=\not \partial \chi, \\
& \not \partial \lambda=\partial \cdot \chi,
\end{aligned}
$$

that link the unconstrained spin- $(s+1 / 2)$ tensor-spinors $\psi$, spin- $(s-1 / 2)$ tensor-spinors $\chi$ and spin- $(s-3 / 2)$ tensor-spinors $\lambda$, are invariant under the unconstrained gauge transformations

$$
\begin{aligned}
& \delta \psi=\partial \epsilon, \\
& \delta \chi=\not \partial \epsilon, \\
& \delta \lambda=\partial \cdot \epsilon,
\end{aligned}
$$


and follow from the rather simple Lagrangians

$$
\begin{aligned}
\mathcal{L}= & i \bar{\psi} \not \partial \psi-i \bar{\psi} \partial \chi+i \partial \bar{\chi} \psi-i s \bar{\chi} \not \partial \chi \\
& +i s \bar{\chi} \partial \lambda-i s \partial \bar{\lambda} \chi-i s(s-1) \bar{\lambda} \not \partial \lambda .
\end{aligned}
$$

We recently came to know that actually the system (6.3), in the slightly different form (6.6, first emerged from a study of the lower excitations of the bosonic string in the low-tension limit in the work of A. Bengtsson [12], and played a key in the more recent, interesting BRTS constructions of Pashnev, Tsulaia and others [13], that we shall comment further upon in the next sections. On the other hand, the fermionic system (2.7) does not play directly a role in the superstring, where totally symmetric tensor-spinors are eliminated by the GSO projection, but it does enter the spectrum of type-0 strings [14. Generalizations of these equations to tensors with mixed symmetry can also be obtained as interesting applications of the BRST procedure, as in [13, 2, but for brevity here we shall confine our attention to the simpler case of fully symmetric tensors.

It is important to notice that eqs. (6.3) and (6.7), that we shall henceforth refer to as "triplets", propagate lower spins as well. This can be foreseen rather simply in the lightcone description of the string states, where, say, the physical polarizations for the system (6.3) would correspond to states created by products of transverse string oscillators of the type $\alpha_{-1}^{i_{1}} \cdots \alpha_{-1}^{i_{s}}|0\rangle$, that indeed describe a nested chain of modes of decreasing spins. The simplest non-trivial case of this type, $\alpha_{-1}^{i_{1}} \alpha_{-1}^{i_{2}}|0\rangle$, presents itself at the first massive level of the open bosonic string. In the $\alpha^{\prime} \rightarrow \infty$ limit these states become massless, and while the traceless part of this two-tensor describes massless spin- 2 modes, its trace describes an independent scalar mode. In general, one thus expects that the local system (6.3) propagate independent sets of irreducible modes of spins $s, s-2$, and so on, down to zero or one according to whether $s$ is even or odd.

In order to analyze directly the physical polarizations described by eqs. (5.3), it is convenient to choose the gauge $C=0$, that can be reached solving a wave equation for the gauge parameter $\Lambda$ and reduces the system to a pair of wave equations for $\phi$ and $D$, together with the constraint

$$
\partial \cdot \phi-\partial D=0
$$

One can now take for $\phi$ and $D$ two plane waves characterized by the same light-like momentum $p_{+}$, and the result follows if the constraints of eq. (6.10) are combined with residual gauge transformations also described by plane waves of momentum $p_{+}$, that as such do not affect the original gauge choice $C=0$, obtaining

$$
\begin{aligned}
& \delta \phi_{(+)^{k}(a)^{s-k}}=k p_{+} \Lambda_{(+)^{k-1}(a)^{s-k}} \\
& p_{+} \phi_{-(+)^{k}(a)^{s-1-k}}=k p_{+} D_{(+)^{k-1}(a)^{s-1-k}}
\end{aligned}
$$

where $a$ stands collectively for any of the other directions, "-" or "i". Using eq. ([.11), one can eliminate all components of the gauge field $\phi$ with at least a lower "+" index, and then eq. (6.12) kills all other components with no lower "+" indices and at least a lower "-" index, while the other choices of $k$ in eq. ([.12) dispose of the whole of $D$. In conclusion, 
one is left with the transverse components $\phi_{i_{1} \cdots i_{s}}$, that indeed describe the (traceless) polarizations of a spin $s$ field, together will all their traces, that indeed correspond to the chain of spins $s, s-2$, and so on, down to zero or one according to whether $s$ is even or odd. A similar, if more involved, argument, applies to the fermionic system, and shows that, with a spinor-tensor $\psi_{\mu_{1} \cdots \mu_{n}}$ and its $\chi$ and $\lambda$ companions, one is actually propagating all half-integer lower spins, together with the maximum one.

Confining for simplicity our attention to the bosonic system ([.3), we can now show how, after a suitable elimination of the $C$ and $D$ fields, $\phi$ satisfies the non-local equations of [1]. This can be done in two different ways. The first consists in the direct elimination of $\mathrm{C}$ from the divergence of the second of eqs. (6.3), that when combined with the third gives

$$
\square D+\frac{1}{2} \partial \partial \cdot D=\frac{1}{2} \partial \cdot \partial \cdot \phi .
$$

Inverting the differential operator in (5.13) defines a solution for $D$ that, by construction, eliminates all lower-spin modes, and substituting it in the second of (6.3) expresses $C$ in terms of $\phi$. Finally, substituting $C$ in the first of eqs. (6.3) yields a non-local fully gauge invariant equation equivalent to (2.15). This can be seen explicitly for the first few cases, but in general one can find a simpler argument noticing that $\phi$ and $D$ define the chain of lower-spin fields $(n \geq 1)$

$$
\mathcal{W}_{n}=\phi^{[n]}-2 n D^{[n-1]}
$$

Eqs. (6.3) and (6.4) imply that these fields satisfy the chain of inhomogeneous Fronsdal equations

$$
\mathcal{F}\left(\mathcal{W}_{n}\right)=\partial^{2} \mathcal{W}_{n+1}
$$

and transform under gauge transformations as ordinary fields of spin $s-2 n$, i.e.

$$
\delta \mathcal{W}_{n}=\partial \Lambda^{[n]} .
$$

One can now look for solutions of eqs. (6.3) that are free of lower-spin excitations. Naively, this would correspond to eliminating $\mathcal{W}_{1}$, but more naturally one can demand that $\mathcal{W}_{1}$ be pure gauge, letting

$$
\mathcal{W}_{1}=\phi^{\prime}-2 D=\partial \alpha,
$$

where eq. (6.3) implies that a gauge transformation affects $\alpha$ according to

$$
\delta \alpha=\Lambda^{\prime} .
$$

Substituting in eqs. (6.3) then gives the inhomogeneous Fronsdal equation

$$
\mathcal{F}(\phi)=3 \partial^{3} \alpha
$$

a local form in terms of the source $\alpha$ of the non-local geometric equations of [1 1 , as advertised. These can be recovered deriving from (6.19) corresponding equations for the $\mathcal{F}^{(n)}$,

$$
\mathcal{F}^{(n)}=(2 n+1) \frac{\partial^{2 n+1}}{\square^{n}} \alpha^{[n]},
$$


where by construction $\alpha$ is replaced by its multiple traces, and thus disappears altogether for $n$ large enough, so that eventually one retrieves the non-local homogeneous equations of section 2. On the other hand, the direct elimination of $\mathcal{W}_{1}$ leads to the usual homogeneous Fronsdal equation with a traceless gauge parameter, but, as is section 5, with an unconstrained gauge potential.

\section{Local compensator forms of the field equations in flat space}

In the previous section we have seen how a single spin- $s$ field can be described by a "triplet" whose lower-spin excitations are restricted to be pure gauge by eq. (6.17), a choice motivated by the fact that $\varphi^{\prime}-2 D$ transforms as a canonical spin- $(s-2)$ field. This indeed turns the first of eqs. (6.6) into (6.19), where $\alpha$ is a spin- $(s-3)$ field that plays the role of the single compensator needed in this formulation, but the second eq. (6.6) takes an apparently more complicated form, and becomes

$$
\square \varphi^{\prime}+\frac{1}{2} \partial \partial \cdot \varphi^{\prime}-\partial \cdot \partial \cdot \varphi=\frac{3}{2} \square \partial \alpha+\partial^{2} \partial \cdot \alpha .
$$

In terms of the Fronsdal operator, however, this simplifies considerably, since eq. (1.1) indeed implies that

$$
\mathcal{F}^{\prime}=2 \square \varphi^{\prime}-2 \partial \cdot \partial \cdot \varphi+\partial \partial \cdot \varphi^{\prime}+\partial^{2} \varphi^{\prime \prime},
$$

so that (7.1) is equivalent to

$$
\mathcal{F}^{\prime}-\partial^{2} \varphi^{\prime \prime}=3 \square \partial \alpha+2 \partial^{2} \partial \cdot \alpha .
$$

On the other hand, the trace of eq. (6.19) is

$$
\mathcal{F}^{\prime}=3 \square \partial \alpha+6 \partial^{2} \partial \cdot \alpha+3 \partial^{3} \alpha^{\prime},
$$

and thus, by comparison, one obtains

$$
\partial^{2} \varphi^{\prime \prime}=4 \partial^{2} \partial \cdot \alpha+\partial^{3} \alpha^{\prime}=\partial^{2}\left(4 \partial \cdot \alpha+\partial \alpha^{\prime}\right) .
$$

The conclusion is that the "triplet equations" (6.3) imply a pair of local equations for a single massless spin- $s$ gauge field $\varphi$ and a single spin- $(s-3)$ compensator $\alpha$,

$$
\begin{aligned}
& \mathcal{F}=3 \partial^{3} \alpha, \\
& \varphi^{\prime \prime}=4 \partial \cdot \alpha+\partial \alpha^{\prime},
\end{aligned}
$$

that are invariant under the unconstrained gauge transformations

$$
\begin{aligned}
& \delta \varphi=\partial \Lambda, \\
& \delta \alpha=\Lambda^{\prime},
\end{aligned}
$$

and clearly reduce to the standard Fronsdal form after a partial gauge fixing using the trace $\Lambda^{\prime}$ of the gauge parameter. 
These local equations are nicely consistent, since the second is implied by the first, as can be seen using the Bianchi identity of eq. (1.14). However, it is important to stress that these are not Lagrangian equations, somewhat in the spirit of the Vasiliev form of higherspin dynamics [7, 8]. A Lagrangian form for the compensator equations can be obtained, as in [13, 2], at the expense of introducing a number of additional fields, although the resulting procedure is nicely guided by the BRST approach.

One can also repeat the construction of local compensator equations for Fermi fields. Here the story parallels the discussion in subsection 3, since the fermionic Fang-Fronsdal operator (3.1) varies into a term proportional to the gamma-trace of the gauge parameter,

$$
\delta \mathcal{S}=-2 i \partial^{2} \notin
$$

under the gauge transformation

$$
\delta \psi=\partial \epsilon
$$

In addition, $\mathcal{S}$ satisfies the "anomalous" Bianchi identity (3.4), and as a result the gauge parameter and the gauge field were constrained in $[5$ to satisfy the conditions

$$
\notin=0, \quad \psi^{\prime}=0 \text {. }
$$

As for integer-spin fields, one can also eliminate these constraints introducing a single compensator field $\xi$. The resulting equations,

$$
\begin{aligned}
& \mathcal{S}=-2 i \partial^{2} \xi, \\
& \psi^{\prime}=2 \partial \cdot \xi+\partial \xi^{\prime}+\not \partial \not ̈,
\end{aligned}
$$

are then invariant under the gauge transformations

$$
\begin{aligned}
& \delta \psi=\partial \epsilon, \\
& \delta \xi=\notin,
\end{aligned}
$$

involving an unconstrained gauge parameter, and are nicely consistent, since the first implies the second via the Bianchi identity (3.4). As in the bosonic case, however, these are not Lagrangian equations. Corresponding Lagrangian equations have not appeared yet, but they are expected to follow from a direct, if tedious, procedure, along the lines of [13].

\section{Local compensator forms of the field equations in (A)dS}

One can also extend the spin- $s$ compensator equations of the previous section to (anti)de Sitter backgrounds, henceforth denoted by (A)dS for brevity. This was done in [2, and it is worthwhile to review briefly these results, given also the central role of (A)dS backgrounds in the Vasiliev formulation [8]. While [13] contains also derivations of Lagrangian equations based on the BRST formalism, here we shall simply content ourselves with an account of the direct constructions of [2]. 
To this end, one needs only two additional inputs, the commutator of two covariant derivatives on a vector

$$
\left[\nabla_{\mu}, \nabla_{\nu}\right] V_{\rho}=\frac{1}{L^{2}}\left(g_{\nu \rho} V_{\mu}-g_{\mu \rho} V_{\nu}\right)
$$

where $L$ determines the AdS curvature and the dS case is recovered formally continuing $L$ to imaginary values, and the corresponding commutator on a spinor,

$$
\left[\nabla_{\mu}, \nabla_{\nu}\right] \eta=-\frac{1}{2 L^{2}} \gamma_{\mu \nu} \eta
$$

where $\gamma_{\mu \nu}$ is antisymmetric in $\mu$ and $\nu$ and equals the product $\gamma_{\mu} \gamma_{\nu}$ when $\mu$ and $\nu$ are different, since these determine all other cases.

Let us therefore begin by considering the bosonic case, noticing that the gauge transformations for the fields $\varphi$ and $\alpha$ in such a curved background take naturally the form

$$
\begin{aligned}
& \delta \varphi=\nabla \Lambda, \\
& \delta \alpha=\Lambda^{\prime},
\end{aligned}
$$

where $\nabla$ denotes and (A)dS covariant derivative.

One can then proceed in various ways, for instance starting from the gauge variation of the (A)dS form of the Fronsdal operator

$$
\begin{aligned}
\delta \mathcal{F}_{L} & \equiv \delta\left\{\mathcal{F}-\frac{1}{L^{2}}[(3-\mathcal{D}-s)(2-s)-s] \varphi-2 g \varphi^{\prime}\right\} \\
& =3\left(\nabla^{3} \Lambda^{\prime}\right)-\frac{4}{L^{2}} g \nabla \Lambda^{\prime}
\end{aligned}
$$

so that

$$
\mathcal{F}_{L}=\mathcal{F}-\frac{1}{L^{2}}\left\{[(3-\mathcal{D}-s)(2-s)-s] \varphi+2 g \varphi^{\prime}\right\},
$$

and it is then simple to conclude that the compensator form of the higher-spin equations in $(\mathrm{A}) \mathrm{dS}$ is

$$
\begin{aligned}
\mathcal{F} & =3 \nabla^{3} \alpha+\frac{1}{L^{2}}\left\{[(3-\mathcal{D}-s)(2-s)-s] \varphi+2 g \varphi^{\prime}\right\}-\frac{4}{L^{2}} g \nabla \alpha, \\
\varphi^{\prime \prime} & =4 \nabla \cdot \alpha+\nabla \alpha^{\prime}
\end{aligned}
$$

These are again nicely consistent, as can be seen making use of the Bianchi identity, that now becomes

$$
\nabla \cdot \mathcal{F}_{L}-\frac{1}{2} \nabla \mathcal{F}_{L}^{\prime}=-\frac{3}{2} \nabla^{3} \varphi^{\prime \prime}+\frac{2}{L^{2}} g \nabla \varphi^{\prime \prime}
$$

and one can in fact verify that the first of (8.6) implies the second.

The fermionic compensator equations (7.12) can be also generalized to (A)dS backgrounds. The gauge transformation for a spin- $s$ fermion becomes in this case

$$
\delta \psi=\nabla \epsilon+\frac{1}{2 L} \gamma \epsilon
$$


where $\nabla$ denotes again an (A)dS covariant derivative, and in order to proceed, one needs only the commutator of two covariant derivatives on a spin- $1 / 2$ field of eq. (8.2).

For a spin- $s$ fermion $\left(s=n+\frac{1}{2}\right)$, where $n$ is the number of vector indices carried by the field $\psi$, the compensator equations in an (A)dS background are then

$$
\begin{aligned}
&(\nabla \psi-\nabla \psi)+ \frac{1}{2 L}[\mathcal{D}+2(n-2)] \psi+\frac{1}{2 L} \gamma \psi \\
&=-\{\nabla, \nabla\} \xi+\frac{1}{L} \gamma \nabla \xi+\frac{3}{2 L^{2}} g \xi \\
& \psi^{\prime}=2 \nabla \cdot \xi+\nabla \not+\nabla \xi^{\prime}+\frac{1}{2 L}[\mathcal{D}+2(n-2)] \not ु-\frac{1}{2 L} \gamma \xi^{\prime}
\end{aligned}
$$

and are invariant under

$$
\begin{aligned}
& \delta \psi=\nabla \epsilon, \\
& \delta \xi=\notin,
\end{aligned}
$$

with an unconstrained parameter $\epsilon$.

Eqs. (8.9) are again non-Lagrangian, like their flat-space counterparts (7.12), but are again nicely consistent, on account of the (A)dS deformation of the Bianchi identity (3.12),

$$
\begin{aligned}
\nabla \cdot \mathcal{S}-\frac{1}{2} \nabla \mathcal{S}^{\prime}-\frac{1}{2} \nabla \boldsymbol{\delta} & =\frac{i}{4 L} \gamma S^{\prime}+\frac{i}{4 L}[(\mathcal{D}-2)+2(n-1)] \phi \\
& +\frac{i}{2}\left[\{\nabla, \nabla\}-\frac{1}{L} \gamma \nabla-\frac{3}{2 L^{2}}\right] \psi^{\prime},
\end{aligned}
$$

where now the Fang-Fronsdal operator $\mathcal{S}$ is also deformed and becomes

$$
\mathcal{S}=i(\nabla \psi-\nabla \psi)+\frac{i}{2 L}[\mathcal{D}+2(n-2)] \psi+\frac{i}{2 L} \gamma \psi
$$

\section{Conclusions}

It is becoming increasingly clear that a deeper understanding of String Theory cannot forego a closer look at its massive excitations. As a result, Higher Spin Gauge Theory is quickly coming to the forefront of current research, since it appears to provide key examples of dynamical systems whose structural complexity is somehow intermediate between those of String Theory and of ordinary low-spin theories. With this is mind, in [1] we reconsidered the standard free higher-spin equations, exhibiting a geometric structure that makes them more akin to their low-spin counterparts. This rests on the replacement, for spin greater than two, of the ordinary local kinetic operators of [0] with non-local ones that guarantee the invariance of the field equations under gauge transformations with unconstrained parameters and the absence in the Bianchi identities of "anomalous" terms depending on the double traces (or triple $\gamma$-traces) of the gauge fields. The end result can be linked to the generalized curvatures introduced by de Wit and Freedman [16], as the local Fronsdal equations were linked in [16] to the traces of the second-order connections. Suitable compensators can dispose of the non-local terms, while preserving the unconstrained gauge 
invariance, but only at the price of making the geometry that underlies the equations less transparent, and therefore they were only briefly discussed in [1].

In [1] we had also noticed that the geometric equations can always be turned to the local, Fronsdal form after partial gauge fixings involving the traces of the gauge parameters. Here we have supplemented this result, showing that if the usual de Donder gauge conditions are modified in order that they be identically traceless, as demanded by the presence of unconstrained fields, the resulting field equations set to zero their double traces on shell. Once these are removed, the study of on-shell polarizations proceeds as in the standard case, despite the apparent initial presence of more field components together with the same number of traceless gauge parameters.

Finally, we have shown how String Theory makes a full, if indirect, use, of the curvatures of [16]. In the bosonic case, these are embodied in a set of local gauge invariant equations propagating modes of $\operatorname{spin} s, s-2$, and so on, down to zero or one according to whether $s$ is even or odd, that follow from the Stueckelberg-like equations for massive string modes in the limit $\alpha^{\prime} \rightarrow \infty$. As we have seen, these reduce to the non-local equations of [1] once one restricts the attention to fields that are free of lower-spin excitations.

The string-inspired systems of "triplets", however, are of interest in their own right, can be simply extended [2] to the mixed-symmetry tensors discussed in [18], and their ability to describe nested chains of lower spins may well prove valuable in establishing more direct links between String Theory and Higher-Spin Gauge Theory, or in providing level-like truncations for the latter. For the time being, following [2], we have seen how the "triplet" equations of section 6 provide an interesting route toward simple non-Lagrangian local compensator forms of the field equations for higher-spin bosons and fermions, that we have displayed both for a flat and an (A)dS background, that plays a key role in the Vasiliev formulation [8]. These display the same unconstrained gauge symmetry as the non-local equations of the previous sections, and are also nicely determined by the BRST approach of [13, 9]. More details, together with local Lagrangian forms for the bosonic equations, are discussed at length in [2].

It will be very interesting to elucidate the possible role of the higher-spin geometry reviewed here in the Vasiliev formulation [8, or in the conformal higher-spin theory proposed by Segal in [19, also in view of its relation to String Theory and to the BRST-like systems studied in [20, 13]. We hope to return to some of these issues in the near future.

\section{Acknowledgments}

It is a pleasure to thank M. Bianchi, G. Pradisi and especially A. Segal for stimulating conversations. This work was supported in part by I.N.F.N., by the EC contract HPRNCT-2000-00122, by the EC contract HPRN-CT-2000-00148, by the INTAS contracts 99-1590 and 03-51-6346, by the MURST-COFIN contracts 2001-025492 and 2003-023852, and by the NATO Contract PST.CLG.978785. Finally, A.S. would like to thank M. Tsulaia for a stimulating collaboration on the topics reviewed in the last sections. 


\section{References}

[1] D. Francia and A. Sagnotti, Phys. Lett. B543 (2002) 303 [arXiv:hep-th/0207002].

[2] A. Sagnotti and M. Tsulaia, Nucl. Phys. B682 (2004) 83, [arXiv:hep-th/0311257].

[3] A. Sagnotti and A. Sevrin, arXiv:hep-ex/0209011.

[4] C. Angelantonj and A. Sagnotti, Phys. Rept. 371 (2002) 1 [arXiv:hep-th/0204089].

[5] C. Fronsdal, Phys. Rev. D18 (1978) 3624; T. Curtright, Phys. Lett. B85 (1979) 219; J. Fang and C. Fronsdal, Phys. Rev. D18 (1978) 3630.

[6] L. P. Singh and C. R. Hagen, Phys. Rev. D9 (1974) 898, Phys. Rev. D9 (1974) 910.

[7] E. S. Fradkin and M. A. Vasiliev, Nucl. Phys. B291 (1987) 141, Annals Phys. 177 (1987) 63, Phys. Lett. B189 (1987) 89, JETP Lett. 44 (1986) 622 [Pisma Zh. Eksp. Teor. Fiz. 44 (1986) 484], Int. J. Mod. Phys. A3 (1988) 2983.

Recent extensions to higher dimensions are discussed in: E. Sezgin and P. Sundell, arXiv:hep-th/0205131, arXiv:hep-th/0205132, arXiv:hep-th/0112100, supergravity," JHEP 0109 (2001) 025 [arXiv:hep-th/0107186].

[8] M. A. Vasiliev, Phys. Lett. B243 (1990) 378, Class. Quant. Grav. 8 (1991) 1387, Phys. Lett. B257 (1991) 111, Phys. Lett. B285 (1992) 225.

For reviews see: M. A. Vasiliev, Int. J. Mod. Phys. D5 (1996) 763 [arXiv:hep-th/9611024], arXiv:hep-th/9910096; arXiv:hep-th/0104246.

[9] W. Siegel and B. Zwiebach, Nucl. Phys. B263 (1986) 105; T. Banks and M. E. Peskin, Nucl. Phys. B264 (1986) 513. For a review see, for instance, W. Siegel, arXiv:hep-th/0107094.

[10] E. Witten, Nucl. Phys. B268 (1986) 253.

[11] M. Kato and K. Ogawa, Nucl. Phys. B212 (1983) 443.

[12] A. K. H. Bengtsson, Phys. Lett. B 182 (1986) 321.

[13] A. Pashnev and M. M. Tsulaia, Mod. Phys. Lett. A 12 (1997) 861 [arXiv:hep-th/9703010];

C. Burdik, A. Pashnev and M. Tsulaia, Nucl. Phys. Proc. Suppl. 102 (2001) 285

[arXiv:hep-th/0103143]; A. Pashnev and M. Tsulaia, Mod. Phys. Lett. A 13 (1998) 1853 [arXiv:hep-th/9803207]; C. Burdik, A. Pashnev and M. Tsulaia, Mod. Phys. Lett. A 16 (2001) 731 [arXiv:hep-th/0101201].

[14] L. J. Dixon and J. A. Harvey, Nucl. Phys. B 274 (1986) 93; N. Seiberg and E. Witten, Nucl. Phys. B 276 (1986) 272; M. Bianchi and A. Sagnotti, Phys. Lett. B 247 (1990) 517;

A. Sagnotti, arXiv:hep-th/9509080, Nucl. Phys. Proc. Suppl. 56B (1997) 332

[arXiv:hep-th/9702093]; C. Angelantonj, Phys. Lett. B 444 (1998) 309

[arXiv:hep-th/9810214]; R. Blumenhagen, A. Font and D. Lust, Nucl. Phys. B 558 (1999) 159 [arXiv:hep-th/9904069]. For a review see 四.

[15] V. Bargmann and I. T. Todorov, J. Math. Phys. 18 (1977) 1141.

[16] B. de Wit and D. Z. Freedman, Phys. Rev. D21 (1980) 358; T. Damour and S. Deser, Annales Poincare Phys. Theor. 47 (1987) 277.

[17] M. Dubois-Violette and M. Henneaux, Lett. Math. Phys. 49 (1999) 245 [arXiv:math.qa/9907135], arXiv:math.qa/0110088.

[18] X. Bekaert and N. Boulanger, arXiv:hep-th/0208058. 
[19] A. Y. Segal, arXiv:hep-th/0207212.

[20] V. E. Lopatin and M. A. Vasiliev, Mod. Phys. Lett. A3 (1988) 257; M. A. Vasiliev, Nucl. Phys. B301 (1988) 26; S. Deser and A. Waldron, Nucl. Phys. B607 (2001) 577 [arXiv:hep-th/0103198]; I. L. Buchbinder, A. Pashnev and M. Tsulaia, Phys. Lett. B523 (2001) 338 [arXiv:hep-th/0109067], and references therein. 